\title{
A high-efficiency resonant cavity for measurement of a beam quadrupole moment
}

\author{
N. Barov, J.S. Kim, FAR-TECH, Inc., San Diego, CA, 92121 \\ C.D. Nantista, R.H. Miller, Stanford Linear Accelerator Center, Menlo Park, CA, 94309
}

Many particle accelerator applications can benefit from online pulse-by-pulse nonintercepting emittance measurement system. Recently, there has been much interest in performing such a measurement with a set of resonant quadrupole-mode cavities. This article explores a geometry to achieve an enhanced shunt impedance in such a cavity by adding a set of posts forming capacitive gaps near the beam pipe outer radius. For typical diagnostic cavity applications, a five-fold increase in shunt impedance can be obtained with this method. The effect of errors in cavity fabrication on the required mode structure are explored.

\section{INTRODUCTION}

The method of measuring emittance by extracting the beam quadrupole moment from a set of stripline beam position monitors (BPM's) was developed by Miller et al. ${ }^{1}$ Whittim and Kolomensky ${ }^{2}$, and Kim et $a .^{3}$ considered the use of a resonant cavity to measure the beam quadrupole moment. This method has similar advantages as using a dipole-mode cavity to measure beam position, namely that the measurement is performed by direct interaction with the cavity mode without having to subtract several large signals as in the case of stripline or button-style pickups. A quadrupole-mode cavity measures $\left\langle x^{2}-y^{2}\right\rangle=\sigma_{x}^{2}-\sigma_{y}^{2}+\langle x\rangle^{2}-\langle y\rangle^{2}$, where the angle brackets are an average over the particle distribution, and $\sigma_{x}$ and $\sigma_{y}$ are the RMS transverse bunch sizes. Nearby dipole cavities measuring $\langle x\rangle$ and $\langle y\rangle$ can be used to subtract the two rightmost terms from this expression in order to give a measurement of $\sigma_{x}^{2}-\sigma_{y}^{2}$. In the absence of beam coupling between the $x$ and $y$ phase spaces, an emittance measurement can be performed by measuring the quadrupole moment at six locations along the beamline at different values of betatron phase advance. ${ }^{1}$ Additionally, a cavity can be tilted by 45 degrees to measure $\langle x y\rangle$, which can be used to diagnose and correct coupling between the $x$ and $y$ beam dimensions.

Quadrupole-mode signals are typically much weaker signal than dipole-mode ones. In order to make accurate measurements of low-emittance high-energy beams, the measurement cavity should be optimized as much as possible. One way to improve measurement sensitivity is to use a multi-cell standing-wave cavity, as is done in the article by Kim et al., ${ }^{4}$ using a 9-cell structure. In this study, the shunt impedance as a function of beam offsets $x$ and $y$ is approximately $R \simeq 800\left(x^{2}-y^{2}\right)^{2} \Omega$, where $x$ and $y$ are in units of millimeters. We use the linac definition of the shunt impedance, with $R=V^{2} / P$, where $V$ is the voltage gained by a relativistic particle crossing the cavity. For a high-current train of pulses such as the Next Linear Collider (NLC) design, such a diagnostic can adequately resolve the quadrupole moment of a beam with $\sigma_{x}=1 \mu \mathrm{m}$, and $\sigma_{y} \ll \sigma_{x}$. In order to make an accurate measurement in this case, the beam should be 
relatively close to the cavity axis, within a few microns.

Multi-cell structures are, however, more difficult to fabricate and tune. In order to obtain a good shunt impedance for the mode, the structure is typically designed to operate in the $\pi$-mode. However, improper cell-to-cell transverse alignment can couple power to all modes in the quadrupole band, with phase advance ranging from 0 to $\pi .^{5}$ This power must eventually be filtered out, which is harder to do in the case of small inter-mode spacing.

In this article, we consider a geometry in which posts extend from the upstream and downstream cavity walls to form four capacitive gaps near the beam pipe diameter. A cavity geometry similar to the one discussed in this article was considered by Leemann and $\mathrm{Yao}^{6}$ for the purpose of making a $500 \mathrm{MHz}$ dipole mode deflection cavity. Their geometry essentially consisted of putting two quarter-wave resonators side-by side. This cavity design had a shunt impedance enhancement of order 100. This paper suggests that such a design can also be used for the purpose of making a BPM cavity. However, when the design is applied to a high-frequency $(>8 \mathrm{GHz})$ BPM cavity with a sufficiently large beam pipe $(>1 \mathrm{~cm})$, the improvement in shunt impedance is only around $40 \%$. This drop in efficiency is mostly due to the rapid scaling of shunt impedance with beam pipe diameter.

A concern in the Leemann and Yao study was the sensitivity of cavity resonant frequency and mode center on the internal rod length. In the case of a quadrupole cavity with four gaps, errors in rod length and placement can result in frequency shift, mode translation, as well as a baseline (monopole-like) shift in the mode pattern. The mode sensitivity to cavity geometry is explored in the section on fabrication tolerances.

\section{SHUNT IMPEDANCE ENHANCEMENT IN A QUADRUPOLE-MODE CAVITY}

In this section we will consider a quadrupole geometry of the type shown in Figure 1. We will assume a beam pipe radius of $a=0.5 \mathrm{~cm}$, and a resonant frequency of $11.424 \mathrm{GHz}$. Although it is possible to write down analytical expressions of the cavity fields in the case of no rods (simple pillbox), or in the case where the rods are much longer than the transverse cavity dimension (Leemann and Yao geometry), our geometry is in an intermediate regime where the analysis will be performed numerically using HFSS. $^{7}$ The cavity shunt impedance will be optimized by varying the height and diameter of the posts, and the diameter and length of the cavity. Although we have examined the case of a round cavity, the outer walls can also be made square or octagonal. This has an influence on the frequencies of the remaining cavity modes. The geometry used in the simulation included a 45 degree chamfer on the sharp edges at the beam pipe and at the ends of the posts.

For the purpose of determining the optimum post height, we consider a cavity with length $l=1.0 \mathrm{~cm}$, and post diameter $d=3 \mathrm{~mm}$. For each value of the post height $h$, the cavity outer wall is adjusted so that the quadrupole mode resonant frequency is maintained at $11.424 \mathrm{GHz}$. As Figure 2 indicates, the shunt impedance rises quickly as a function of $h$ until $h=3.2 \mathrm{~mm}$, and then rapidly drops. Cases with $h>3.5 \mathrm{~mm}$ correspond to cavity geometries where the cavity diameter $b$ is too small with $b<1.26 \mathrm{~cm}$. The maximum shunt impedance is 5.3 times larger than that for a bare cavity with $h=0$, while the maximum $R / Q$ value is enhanced by a factor up to 11.5 . 
Next, we examine the effect on shunt impedance as a result of the choice of post diameter $d$. For each diameter in Figure 3, the optimum shunt impedance occurs at a different value of the post height. The outer diameter of each cavity configuration was again adjusted to reach the target $11.424 \mathrm{GHz}$ resonance frequency. Although the $2 \mathrm{~mm}$ diameter posts outperform the $3 \mathrm{~mm}$ ones in shunt impedance by about $5 \%$, we prefer the larger diameter because we envision the structure to be easier to fabricate. For the remainder of this article, we will consider only the case of $3 \mathrm{~mm}$ posts.

The shunt impedance can further be optimized by adjusting the cavity length. The shunt impedance at each value of cavity length is optimum near the same value of the cavity outer radius, so the simulations were performed at a fixed outer radius of $b=1.77$ $\mathrm{cm}$, and the cavity frequency was corrected by altering the height of the posts. Figure 5 shows that the optimum point is for a slightly longer cavity of $1.1 \mathrm{~cm}$.

The posts in our design locally concentrate the field so that it deviates from a pure quadrupole pattern. The field components that are allowed by symmetry above a quadrupole are described by m-numbers of $\mathrm{m}=6,10,14$, etc., where field dependence is $e^{i m \phi}$. In order to accurately determine the coefficients of those terms, $V$ has been plotted as a function of the azimuthal angle far off-axis, at an offset of $4.5 \mathrm{~mm}$ (see Figure 4). The cavity voltage for $\mathrm{y}=0$, normalized to a unity quadrupole component is then,

$$
V=x^{2}+1.57 \times 10^{-4} x^{6}+5.56 \times 10^{-8} x^{10}
$$

with $x$ measured in $\mathrm{mm}$. For a beam confined within a $1 \mathrm{~mm}$ radius, which will almost always be the case for this type of diagnostic, these higher order moments are negligible. At the same time, we may justify our choice of a $2.5 \mathrm{~mm}$ offset integration line used to produce the earlier plots, as neglecting the higher-order moments leads to an error of $1 \%$ in the quadrupole shunt impedance.

The performance of a quadrupole-mode cavity is partly determined by the spacing between the desired mode, and the remaining cavity modes. Analysis of a near-square pillbox cavity by Kim et al. ${ }^{3}$ indicates that a combination of $\mathrm{TM}_{310}$ and $\mathrm{TM}_{130}$ modes can couple on-axis. These modes tend to be close in frequency at $12.6 \mathrm{GHz}$ and $13.4 \mathrm{GHz}$, and the tail of the frequency distribution can extend to 11.424 and thus limit resolution. The mode at $13.4 \mathrm{GHz}$ has a monopole-like form near the axis and thus couples strongly to the beam.

For the cavity used for the Figure 4 analyses, the fundamental mode is at $5.6 \mathrm{GHz}$, and the dipole modes are at $8.7 \mathrm{GHz}$. A TE-like mode appears at $13.8 \mathrm{GHz}$, but will not couple for a beam propagating parallel to the cavity axis. The orthogonal quadrupole mode with electric field maxima rotated 45 degrees from the posts is at $14.2 \mathrm{GHz}$. With slightly bigger posts and smaller cavity outer radius, this mode can easily be made to resonate at $>18 \mathrm{GHz}$ if needed. The mode which corresponds to a $\mathrm{TM}_{200}$ mode in a cylindrical cavity occurs at $15 \mathrm{GHz}$. The frequency of this mode can also be increased, if needed.

In this section, we have explored the optimization of shunt impedance and $R / Q$ as a function of several cavity parameters, but with a fixed beam pipe radius. Some further optimization may be possible by applying more rounding to both inside and outside corners, canting the pole faces, and optimizing the cross-section shape for the posts. 


\section{SENSITIVITY TO FABRICATION ERRORS}

The work of Leemann and $\mathrm{Yao}^{6}$ showed that their cavity design was quite sensitive to the length and placement of the rods, which can modify the mode frequency or shift the cavity electrical center. The same is true of a quadrupole mode, with the additional concern that the mode center can be misaligned along both transverse dimensions. In the case of a quadrupole mode, we have also found one additional type of field distortion that adds a base-line shift to the cavity output signal.

Our quadrupole cavity design has a set of four gaps of length $g$ located at azimuthal angles of $0,90,180$ and 270 degrees. In order to find the sensitivity to small variations in gap lengths we have simulated a case with the gaps at 0 and 180 degrees kept at the original size and the remaining two gaps reduced by $\delta g=100 \mu \mathrm{m}$. This results in a cavity shunt impedance near the axis given by:

$$
R(x, y)=505\left(x^{2}-y^{2}+0.069\right)^{2} \Omega,
$$

with an on-axis shunt impedance given by this expression of $R(0,0)=2.44 \Omega$. Thus, a round, centered beam will generate a signal that's many times greater than the detection threshold, which will add noise to the measurement due to the limited dynamic range and precision of the receiver and readout electronics.

In order to establish the tolerance for $\delta g$, we have conducted a series of HFSS runs to study the dependence of the on-axis shunt impedance on this parameter. These results have a good fit to a quadratic,

$$
R(0,0) \propto(\delta g)^{2}
$$

The quantity $R(0,0)$ is also a function of cavity tilt relative to the beam axis. Thus, cavity tilt can be used to compensate for the imperfection in the gap sizes. We have performed an HFSS simulation with both variations in gap sizes and a tilted integration line to simulate the combination of these two effects. This result shows that the case of $\delta g=100 \mu \mathrm{m}$ can be compensated by a 130 mili-radian tilt. Using the scaling of equation (3), a $\delta g$ of $10 \mu \mathrm{m}$ can be compensated by a tilt of 1.3 mili-radians, which is a reasonable amount.

A non-zero on-axis shunt impedance can also be found in a conventional cavity with nearly smooth walls that have only slight imperfections. In order to simulate this case, we consider a cavity where the posts at the 0 and 180 degree positions have been removed, and the posts in the remaining locations have been cut down to a height of $\delta g / 2$. For $\delta g=200 \mu \mathrm{m}$, we find that such a cavity has,

$$
R(0,0)=3.04 \Omega
$$

Although this value for $R(0,0)$ is smaller than an equivalent $\delta g$ in a cavity with $3 \mathrm{~mm}$ long posts, the plain cavity also generates less signal for a given beam quadrupole moment. Because of this, the plain cavity is actually more strongly affected by the imperfections.

In the cavity with the $3.15 \mathrm{~mm}$ long posts, errors in fabrication can lead not only 
to uneven gaps, but also errors in the placement of the posts. We have simulated a case where the posts at 0 and 180 degrees are kept in their original locations, and the posts at 90 and 270 degrees have been moved away from the cavity center by an amount $\delta r$. For $\delta r=50 \mu \mathrm{m}$, the on-axis shunt impedance is,

$$
R(0,0)=6.9 \Omega
$$

Simulations of several cases with $\delta r$ ranging from 25 to $100 \mu \mathrm{m}$ again show a quadratic dependence, $R(0,0) \propto(\delta r)^{2}$. Since in this case $R(0,0)$ is larger than in the case of variations in the height of the posts, the radial placement of the posts must be kept to a somewhat tighter tolerance than the height dimension, by a factor of 1.7.

We have only explored the effects of variations in the post height and radial placement. However, any perturbation in geometry located near the azimuthal angle of one of the longitudinal electric field maxima can have the same effect. This includes perturbations at the edge of the beam pipe and at the cavity outer wall.

A cavity tilted with respect to the beam axis has one additional effect. If the cavity voltage varies in time as $e^{i \omega t}$, the integrated voltage seen by a beam particle may also have a complex component which can be represented as,

$$
V=A\left(x^{2}-y^{2}\right)+i B(x+y)+i C(x-y) .
$$

where $A, B$, and $C$ are constants. The imaginary terms have a dipole pattern whose overall direction is determined by the cavity tilt. A rough estimate of the cavity power due to this is that a beam offset by 0.5 micron in a cavity tilted by 1 mili-radian produces an equivalent signal strength (but rotated 90 degrees in phase) as an on-axis beam with $\sigma_{x}$ $=1 \mu \mathrm{m}$ and $\sigma_{y}=0$. This is a weak signal which can be distinguished from the main signal because it is at orthogonal phase. However, cavity tilts much larger than this can produce stronger signals which are undesirable from the point of view of the processing electronics' limited dynamic range.

\section{EFFECT OF BEAM PIPE DIAMETER}

As the Leemann and $\mathrm{Yao}^{6}$ study indicates, the shunt impedance is a strong function of the transverse separation between the posts. In our geometry, this is constrained by the beam pipe diameter, so we expect the shunt impedance to strongly depend on this quantity. In addition to the $1.0 \mathrm{~cm}$ beam pipe diameter, we have also simulated optimized cases with $8 \mathrm{~mm}$ and $12 \mathrm{~mm}$ diameter, as shown in Figure 6. Under these conditions, the shunt impedance changes by a factor of $\sim 2$ for a $2 \mathrm{~mm}$ change in beam pipe diameter.

We have avoided the more complicated task of evaluating the shunt impedance over a broader range of beam pipe diameters, since that brings up questions of whether the post diameter should also be scaled, and also whether the shunt impedance should be corrected for the presence of higher-moment components.

\section{SEXTUPOLE-MODE CAVITY}


The addition of posts to form capacitive gaps near the the electric field maxima can be used to enhance the shunt impedance of sextupole and higher order modes. A sextupole mode can detect an asymmetric component of the beam distribution. One application is for detecting the presence of a beam tail, for providing an early warning of beam breakup due to short-range wakes in a linac.

We have simulated a cavity geometry with a $1.0 \mathrm{~cm}$ cavity length, a $1.7 \mathrm{~cm}$ outer radius and $3 \mathrm{~mm}$ diameter, $3.0 \mathrm{~mm}$ high posts forming six gaps in each 60 degree interval around the cavity, tangent to the beam pipe radius of $5 \mathrm{~mm}$. The resonant frequency of this cavity is $14.28 \mathrm{GHz}$. The shunt impedance near the axis is given by,

$$
R(x, y)=11.15\left(x^{3}-3 x y^{2}\right)^{2} \Omega
$$

where distances are measured in mm. For the purpose of comparing to a plain cavity with no posts, we use the results from the study of a standing-wave cavity operating in the $3 \pi / 4$-mode. The cell length was $11 \mathrm{~mm}$, the cell longitudinal centers were located 13.1 $\mathrm{mm}$ apart, and the beam pipe radius was $5 \mathrm{~mm}$. The combined shunt impedance for two cells (one live and one dead) was $0.45 \Omega$ at a $1 \mathrm{~mm}$ offset. By comparison, the above result for the cavity with posts is 25 times larger, and has a 70 times larger $R / Q$. These enhancements are quite significant, and can be combined with the use of multiple cavities and further optimization of the beam pipe radius. Such measures can partially overcome the inherently lower sensitivity of a sextupole mode.

\section{DISCUSSION}

We have presented a cavity geometry that offers improved shunt impedance for the measurement of a beam quadrupole, sextupole, and higher order moments. This geometry also has advantages in that the remaining cavity modes can be spaced further apart from the mode of interest. In the case of quadrupole-mode cavities, our proposed geometry requires a fairly tight set of mechanincal tolerances.

${ }^{1}$ R.H. Miller, J.E. Clendenin, M.B. James, J.C. Sheppard, Proc. $12^{\text {th }}$ Int. Conf. On High Energy Acc. (Fermilab, Batiavia, 1983), SLAC-PUB-3186.

${ }^{2}$ D.H. Whittum and Y.K. Kolomensky, Rev. Sci. Instr. 70 (1999) 2300.

${ }^{3}$ J.S. Kim, C.D. Nantista, R.H. Miller, A.W. Weidemann, “A Resonant Cavity Approach to Non-Invasive Pulse-to-Pulse Emittance Measurement", submitted to Rev. Sci. Instr.

${ }^{4}$ J.S. Kim, R.H. Miller, C.D. Nantista, Rev. Sci. Instr. 76, 1 (2005).

${ }^{5}$ N. Barov, J.S. Kim, A.W. Weidemann, R.H. Miller, C.D. Nantista, "High-Precision Resonant Cavity Beam Position, Emittance and Third-Moment Monitors", Proc. Of the 2005 Part. Accel. Conf.

${ }^{6}$ C. Leemann and C.G. Yao, Proceedings of the 1990 Linac conference, p. 232.

${ }^{7}$ HFSS ver. 9.0: Ansoft High Frequency Structure Simulation Code, Ansoft Corporation, Pittsburgh, PA, <http://www.ansoft.com> 
FIG. 1. A 3-D view of the model geometry. The cavity has a beam pipe of radius $a$, an outer radius of $b$, and a length $l$. The eight posts are tangential to the beam pipe radius and have a diameter $d$ and height (or length) $h$. The beam pipe is along the $z$-axis, while one set of posts is offset along the $x$-plane, and the other along the $y$-plane.

FIG. 2. Dependence of $R$ and $R / Q$ on the height of the posts. For each data point, the cavity outer wall radius was adjusted to maintain a resonance frequency of 11.424 $\mathrm{GHz}$. The shunt impedance was computed with an integration line offset by $2.5 \mathrm{~mm}$ from the beam pipe axis.

FIG. 3. Cavity shunt impedance vs. $h$ for a post diameter of $4 \mathrm{~mm}$ (x's), $3 \mathrm{~mm}$ (circles), and $2 \mathrm{~mm}$ (plus sign). The solid lines are fits to a second-degree polynomial. The shunt impedance is measured at a $2.5 \mathrm{~mm}$ offset along the $x$-axis.

FIG. 4. Shunt impedance as a function of azimuthal angle $\phi$ at $4.5 \mathrm{~mm}$ offset from the axis. The form of the fit function is a linear combination of $\cos (2 \phi), \cos (6 \phi)$, and $\cos (10 \phi)$, with a least squares fit of

$$
V=3.70 \cos (2 \phi)-0.239 \cos (6 \phi)+0.035 \cos (10 \phi) \text {. }
$$

FIG. 5. Shunt impedance vs. cavity length. The shunt impedance was calculated at an offset of $2.5 \mathrm{~mm}$ from the axis. In each case, cavity length was $1.0 \mathrm{~cm}$, and the post diameter was $3 \mathrm{~mm}$.

FIG. 6. Dependence of shunt impedance on beam pipe diameter. Each case was optimized with the procedure of Figure 7. The shunt impedance is calculated at an offset of 2.5 $\mathrm{mm}$ from the axis. 


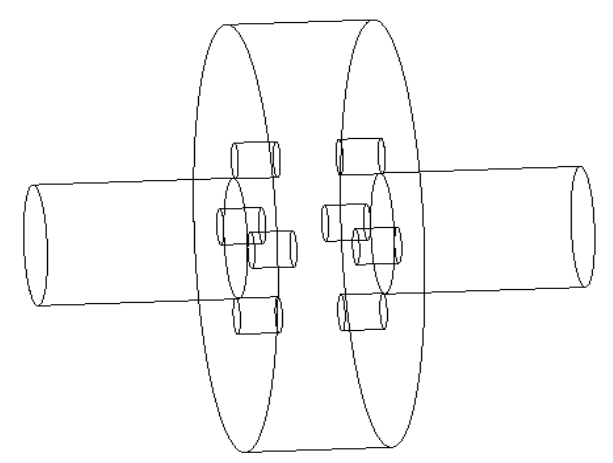




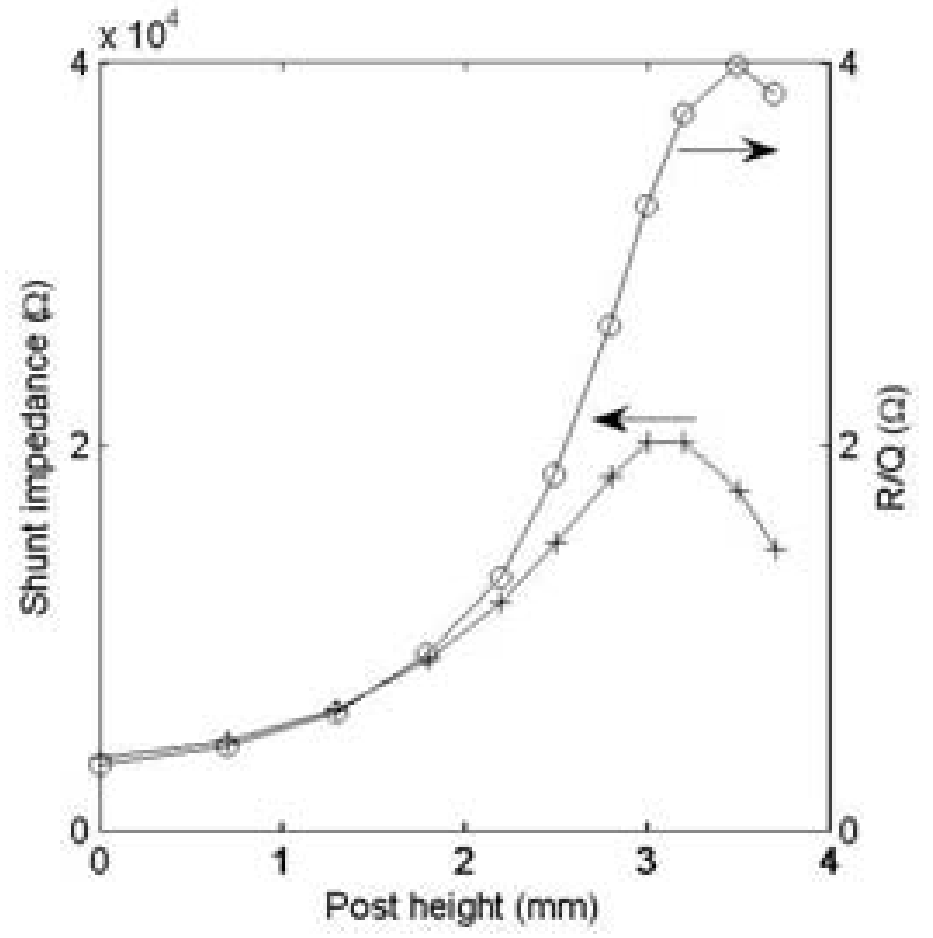




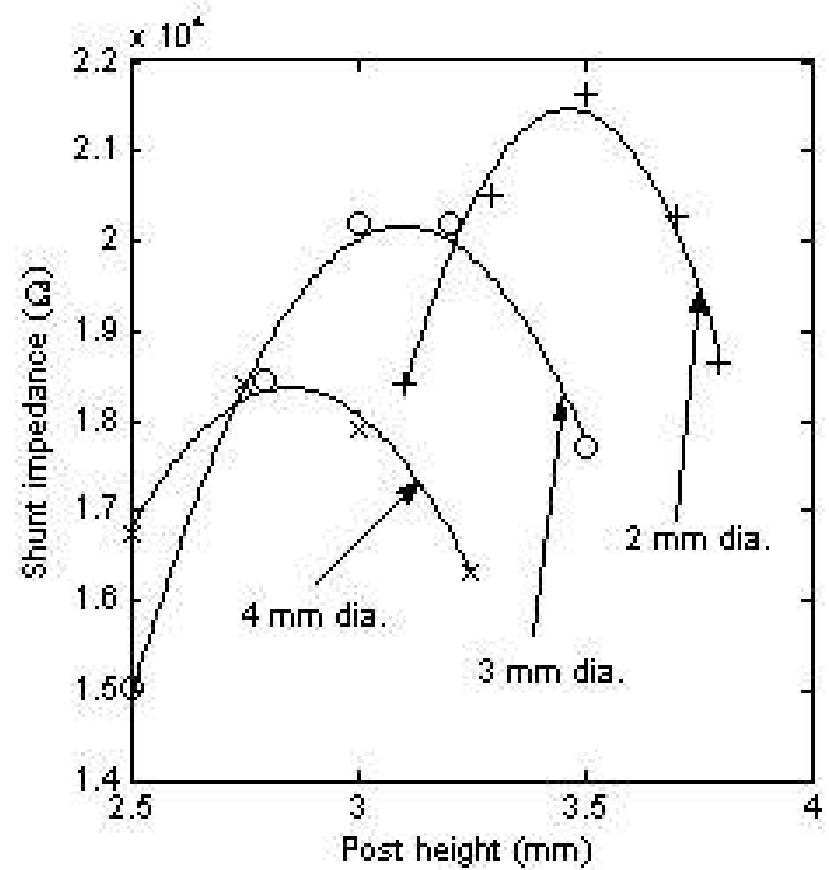




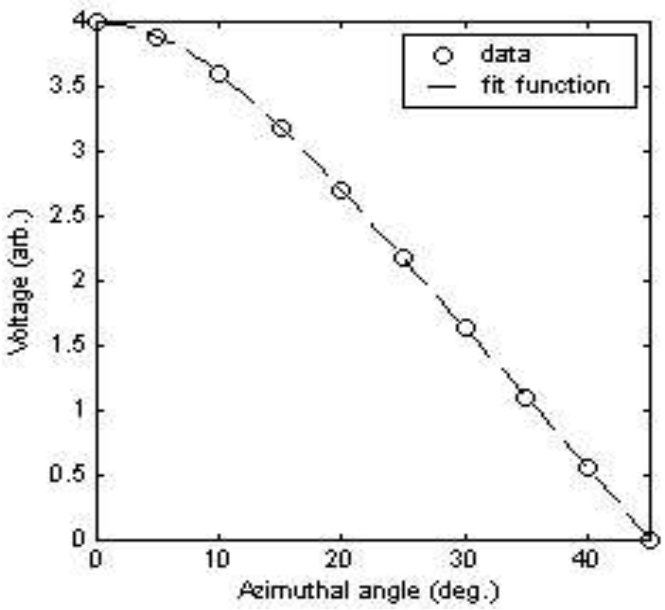




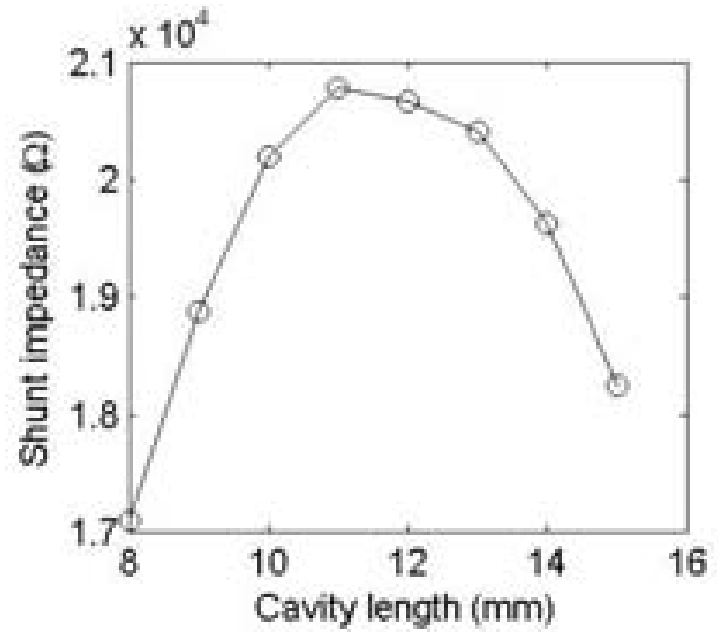




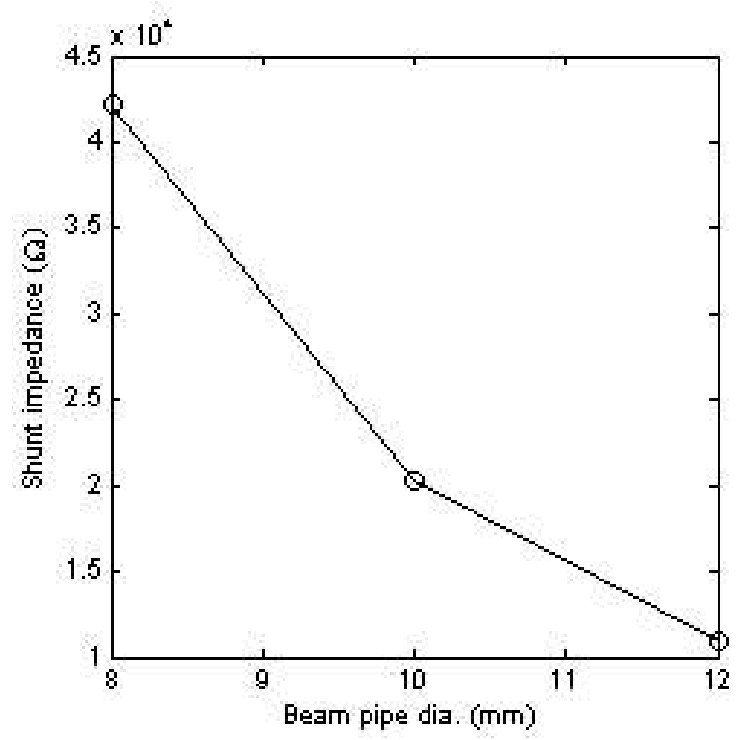

